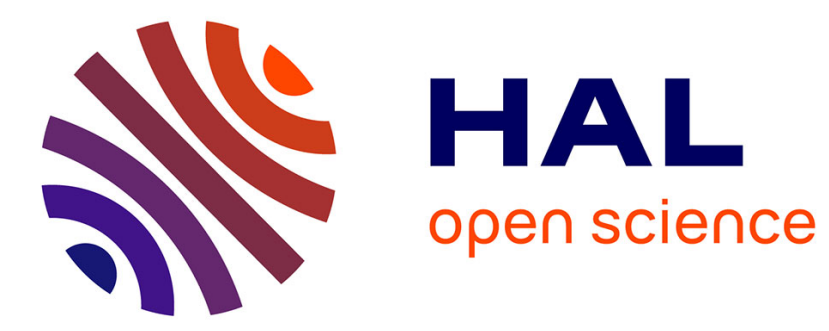

\title{
Homogeneous localizations of some quantum enveloping superalgebras
}

\author{
Jacques Alev, François Dumas
}

\section{To cite this version:}

Jacques Alev, François Dumas. Homogeneous localizations of some quantum enveloping superalgebras. Journal of Algebra and Its Applications, 2021, 20 (1), 2140005, 25 pp. hal-02403008

\section{HAL Id: hal-02403008 \\ https://hal.science/hal-02403008}

Submitted on 10 Dec 2019

HAL is a multi-disciplinary open access archive for the deposit and dissemination of scientific research documents, whether they are published or not. The documents may come from teaching and research institutions in France or abroad, or from public or private research centers.
L'archive ouverte pluridisciplinaire HAL, est destinée au dépôt et à la diffusion de documents scientifiques de niveau recherche, publiés ou non, émanant des établissements d'enseignement et de recherche français ou étrangers, des laboratoires publics ou privés. 


\title{
HOMOGENEOUS LOCALIZATIONS OF SOME QUANTUM ENVELOPING SUPERALGEBRAS
}

\author{
JACQUES ALEV AND FRANÇOIS DUMAS
}

Dedicated to Nicolás Andruskiewitsch on his sixthieth birthday

\begin{abstract}
Under suitable conditions the skewfield of fractions of a superalgebra which is a noetherian domain is canonically provided with a structure of superalgebra. This gives rise to a notion of rational equivalence in the category of superalgebras. We study from the point of view of this rational equivalence some low dimensional examples of quantum enveloping algebras of Lie superalgebras.
\end{abstract}

\section{INTRODUCTION}

The initial question of the Gelfand-Kirillov problem in the seminal article [14] is to determine conditions for the skewfield of fractions of the enveloping algebra of an algebraic finite dimensional Lie algebra to be isomorphic to the skewfield of fractions of a Weyl algebra over a purely transcendental extension of the base field. In the case of quantum enveloping algebras, the algebras of quantum polynomials play the role of Weyl algebras as canonical models in the rational classification. The literature on these topics is very wide, see section I of [17] or section 1 of [13] for some recent surveys and references.

This paper is devoted to a formulation of the Gelfand-Kirillov property in the category of superalgebras. Two general principles emerge from this study. According to the first one, we can extend the superalgebra structure to the skewfield of fractions of the superalgebras under consideration and then place the questions of rational classification or rational separation in terms of isomorphisms of superalgebras. The second one is that such rational isomorphisms, when they exist, are obtained at the level of intermediate localizations (by one element under suitable assumptions) without having to go to the whole skewfield of fractions. The condition of being a domain leads naturally to focus on the case of the enveloping algebras of some orthosymplectic superalgebras and their quantum analogues.

Date: November 22, 2019.

2010 Mathematics Subject Classification. Primary 17B37; Secondary 17B35, 17A70, $16 \mathrm{~S} 85$.

Key words and phrases. Superalgebra, Lie superalgebra, enveloping algebra, quantum superalgebra, Gelfand-Kirillov hypothesis. 
We summarize in the first part some preliminary general results on localization processes in superalgebras that are Noetherian domains. Some of these arguments are more or less implicitly known in the literature (see [6, 15, 25]) but we give here for the reader's convenience self-contained formulations and proofs adapted to the forthcoming applications. In particular, theorem 1.2.2 proves that, for any superalgebra $A$ which is a noetherian domain such that $A$ is finitely generated as a left module over its even subalgebra $A_{0}$, the skewfield of fractions of $A$ is canonically provided with a superalgebra structure. Two superalgebras satisfying these conditions are said to be s-rationally equivalent if their skewfields of fractions are isomorphic as superalgebras. This applies in particular to the enveloping algebras of the finite dimensional Lie superalgebras which are domains, and some significant examples studied in the previous paper [3] from the point of view of the ordinary rational equivalence can be revisited from the finer point of view in the category of superalgebras.

The second and third part are devoted to exploratory examples of quantum enveloping algebras $\mathcal{U}_{q}(\mathfrak{g})$ for $\mathfrak{g}$ a finite dimensional Lie superalgebra. Defining a suitable notion of $q$-polynomial superalgebra, we ask when $\mathcal{U}_{q}(\mathfrak{g})$ is rationally equivalent as a superalgebra to a $q$-polynomial superalgebra over a center which is a purely transcendental extension of the base field $\mathbb{k}$. We prove that the answer is positive for $\mathfrak{g}=\mathfrak{o s p}(1,2)$, for $\mathfrak{g}=\mathfrak{n}$ the nilpotent positive part of $\mathfrak{o s p}(1,4)$, for $\mathfrak{g}=\mathfrak{b}$ the positive Borel subsuperalgebra of $\mathfrak{o s p}(1,4)$, and for $\mathfrak{g}=\mathfrak{p}$ a parabolic subsuperalgebra of $\mathfrak{o s p}(1,4)$. A notable fact is that in all cases isomorphisms are obtained after localization by one element.

In addition, the second section details in the case $\mathfrak{g}=\mathfrak{o s p}(1,2)$ a parallel study of the enveloping algebra $\mathcal{U}(\mathfrak{g})$ and its quantum analogue $\mathcal{U}_{q}(\mathfrak{g})$. We prove in particular that $\mathcal{U}_{q}(\mathfrak{g})$ contains a subalgebra isomorphic to the quantum enveloping algebra $\mathcal{U}_{q}\left(\mathfrak{g}_{0}\right)$ of the even part $\mathfrak{g}_{0}=\mathfrak{s l}(2)$. Then rational separation arguments allow to determine all possible and impossible embeddings between the skewfields of fractions of the four algebras $\mathcal{U}(\mathfrak{s t}(2))$, $\mathcal{U}(\mathfrak{o s p}(1,2)), \mathcal{U}_{q}(\mathfrak{s l}(2))$ and $\mathcal{U}_{q}(\mathfrak{o s p}(1,2))$.

\section{Some PRELIMINARY RESUlts}

Let $\mathbb{k}$ be a commutative field. In this paper, algebra always means associative non necessarily commutative $\mathbb{k}$-algebra with unit. We say that a $\mathbb{k}$-algebra $A$ is an Ore domain if $A$ is a domain and the multiplicative set $S=A \backslash\{0\}$ is a left and right Ore subset in $A$. In this case, we denote by Frac $A=A S^{-1}=S^{-1} A$ the skewfield of fractions of $A$. In particular if $A$ is a noetherian domain, then $A$ is an Ore domain.

1.1. Localization results in noetherian domains. We fix in this subsection a $\mathbb{k}$-algebra $A$ which is a noetherian domain.

Lemma 1.1.1. Let $x$ be an element of $A$ such that the multiplicative subset $X$ of $A$ generated by $x$ is a left and right Ore set in $A$, and denote by $A^{\prime}$ the 
localized algebra $A X^{-1}=X^{-1} A$. Let $B=A[y ; \tau, \delta]$ be an Ore extension of $A$ where $\tau$ is a $\mathbb{k}$-automorphism of $A$ and $\delta$ is a $\tau$-derivation of $A$. We suppose that there exists some element $\lambda \in \mathbb{k}^{\times}$such that $\tau(x)=\lambda x$. Then $\tau$ and $\delta$ extend to $A^{\prime}, X$ is a left and right Ore set in $B$, and the Ore extension $B^{\prime}=A^{\prime}[y ; \tau, \delta]$ identifies with the localized algebra $B X^{-1}=X^{-1} B$.

Proof. Let us recall that $B=A[y ; \tau, \delta]$ is the $\mathbb{k}$-algebra of polynomials $\sum_{i} a_{i} y^{i}, a_{i} \in A$, with product twisted by the commutation relation $y a=$ $\tau(a) y+\delta(a)$ for any $a \in A$. It is well known that $B$ inherits from $A$ the property of being a noetherian domain. In particular the localized subalgebras considered in the lemma can be identifed with subalgebras of Frac $A \subseteq$ Frac $B$.

The unique way to extend $\tau$ and $\delta$ to $A^{\prime}$ is to define $\tau\left(x^{-1}\right)=\lambda^{-1} x^{-1}$ and $\delta\left(x^{-1}\right)=-\tau\left(x^{-1}\right) \delta(x) x^{-1}=-\lambda^{-1} x^{-1} \delta(x) x^{-1}$. Then the commutation rule $y x=\tau(x) y+\delta(x)$ in $B$ implies $x^{-1} y=\lambda y x^{-1}+x^{-1} \delta(x) x^{-1}$ in $B^{\prime}$. We have in $A^{\prime}$ an equality $x^{-1} \delta(x)=a x^{-d}$ for some $a \in A$ and some integer $d \geq 0$, which implies $x^{-1} y=\left(\lambda y x^{d}+a\right) x^{-d-1}$. Then $x^{-2} y=\lambda x^{-1} y x^{-1}+x^{-1} a x^{-d-1}=$ $\lambda\left(\lambda y x^{d}+a\right) x^{-d-2}+a_{1} x^{-d^{\prime}-d-1}=\lambda^{2} y x^{-1}+\lambda a x^{-d-2}+a_{1} x^{-d^{\prime}-d-1}$ for some $a_{1} \in A$ and $d^{\prime} \geq 0$ defined by $x^{-1} a=a_{1} x^{-d^{\prime}}$. It follows that $x^{-2} y=s_{2} x^{-d_{2}}$ for some $s_{2} \in B$ and $d_{2}=\max \left(2, d+2, d^{\prime}+d+1\right) \geq 2$, and inductively $x^{-i} y=s_{i} x^{-d_{i}}$ for some $s_{i} \in B$ and some integer $d_{i} \geq i$ for any $i \geq 1$. We deduce that $x^{-i} y^{j}=t_{i, j} x^{-e_{i, j}}$ for some $t_{i, j} \in B$ and some integer $e_{i, j} \geq i$ for any $i \geq 1, j \geq 0$.

Let $b^{\prime}=\sum_{j=0}^{p} a_{j}^{\prime} y^{j}$ be an element of $B^{\prime}$, with $a_{j}^{\prime} \in A^{\prime}$ for any $0 \leq j \leq p$. For any $0 \leq j \leq p$, we have $a_{j}^{\prime}=x^{-n_{j}} a_{j}$ with $n_{j} \in \mathbb{N}$ and $a_{j} \in A$. We obtain $b^{\prime}=x^{-n} b$ with notations $n=\max \left(n_{j}\right)$ and $b=\sum_{j=0}^{p} x^{n-n_{j}} a_{j} y^{j} \in B$. On the other side, we also have for any $0 \leq j \leq p$ an expression $a_{j}^{\prime}=c_{j} x^{-m_{j}}$ with $m_{j} \in \mathbb{N}$ and $c_{j} \in A$. We denote $m=\max _{0 \leq j \leq p}\left(m_{j}\right)$. If $m=0$, then $b^{\prime} \in B$. Suppose that $m \geq 1$. Then $b^{\prime}=\sum_{j=0}^{p} c_{j} x^{m-m_{j}} x^{-m} y^{j}=$ $\sum_{j=0}^{p} c_{j} x^{m-m_{j}} t_{m, j} x^{-e_{m, j}}$. We introduce $f=\max _{0 \leq j \leq p}\left(e_{m, j}\right)$ in order to obtain $b^{\prime}=c x^{-f}$ where $c \in B$ denotes the sum $\sum_{j=0}^{p} c_{j} x^{m-m_{j}} t_{m, j} x^{f-e_{m, j}}$.

We conclude that, for any $b^{\prime} \in B^{\prime}$, there exist $b, c \in B$ and $m, n \in \mathbb{N}$ such that $b^{\prime}=x^{-n} b=c x^{-m}$. The converse inclusion $X^{-1} B \subseteq B^{\prime}$ is clear and the proof is complete.

Lemma 1.1.2. Let $x, y$ be two elements of $A$ such that $x y=\lambda y x$ for some element $\lambda \in \mathbb{k}^{\times}$. We suppose that the multiplicative subsets $X$ and $Y$ respectively generated by $x$ and $y$ are left and right Ore sets in A. Then:

(i) The multiplicative subset $S_{x, y}$ generated by $x$ and $y$ is a right and left Ore set in $A$.

(ii) The subset $Y$ is a right and left Ore set in the localized algebra $A^{\prime}=$ $A X^{-1}=X^{-1} A$, and the localized algebra $B^{\prime}=A^{\prime} Y^{-1}$ satisfies :

$$
B^{\prime}=A^{\prime} Y^{-1}=A S_{x, y}^{-1}=S_{x, y}^{-1} A=Y^{-1} A^{\prime} .
$$


(iii) The multiplicative subset $Z$ generated by the product $z=x y$ is a right and left Ore set in $A$, and we have $B^{\prime}=A Z^{-1}=Z^{-1} A$.

Proof. It follows obviously from relation $x y=\lambda y x$ that any element of $S_{x, y}$ can be written $x^{n} y^{m}$ or $y^{m} x^{n}$ with $m, n \in \mathbb{N}$ up to multiplication by some power of $\lambda \in \mathbb{k}^{\times}$. We fix $a \in A$ and $(n, m) \in \mathbb{N}^{2}$. Since $Y$ is an Ore set, there exist $c \in A$ and $q \in \mathbb{N}$ such that $y^{q} a=c y^{m}$. Then $X$ being an Ore set, there exist $b \in A$ and $p \in \mathbb{N}$ such that $x^{p} c=b x^{n}$. Finally $x^{p} y^{q} a=b x^{n} y^{m}$ and, with similar calculations on the right, point (i) is proved.

We consider now an integer $m \in \mathbb{N}$ and an element $a^{\prime}=a x^{-n} \in A^{\prime}$ with $n \in \mathbb{N}$. By point (i), there exist $b \in A$ and $(p, q) \in \mathbb{N}^{2}$ such that $x^{p} y^{q} a=b x^{n} y^{m}=b \lambda^{m n} y^{m} x^{n}$. Then $y^{q}\left(a x^{-n}\right)=\left(x^{-p} \lambda^{m n} b\right) y^{m}$ with $a x^{-n}$ and $x^{-p} \lambda^{m n} b$ in $A^{\prime}$. With similar calculations on the right, we conclude that $Y$ is a left and right Ore set in $A^{\prime}$. The last equalities in point (ii) are clear by the relation $x y=\lambda y x$.

Denoting $z=x y$, we have $z^{n}=\lambda^{-n(n-1) / 2} x^{n} y^{n}=\lambda^{n(n+1) / 2} y^{n} x^{n}$ for any $n \in \mathbb{N}$. We fix $a \in A$ and $n \in \mathbb{N}$. Aplying point (i) with $m=n$, there exist $b \in A$ and $(p, q) \in \mathbb{N}^{2}$ such that $y^{q} x^{p} a=b z^{n}$. If $q \leq p$, this implies $y^{p} x^{p} a=y^{p-q} b z^{n}$ and then $z^{p} a=c z^{-n}$ with $c=\lambda^{p(p+1) / 2} y^{p-q} b \in A$. If $p \leq q$, we transform $y^{q} x^{p} a=\lambda^{-p q} x^{p} y^{q}=\lambda^{-p q} x^{p-q} x^{q} y^{q}$ and deduce that $z^{q} a=d z^{-n}$ with $d=\lambda^{p q-q(q+1) / 2} x^{p-q} \in A$. With similar calculations on the right, we conclude that $Z$ is a left and right Ore set in $A$. It is clear that $A Z^{-1} \subseteq A S_{x, y}^{-1}$. The above calculation proves that conversely any fraction $a\left(x^{n} y^{m}\right)^{-1}$ with $a \in A$ and $(n, m) \in \mathbb{N}^{2}$ can be rewritten as $b z^{-k}$ with $b \in A$ and $k=\min (m, n)$, and the proof is complete.

Corollary 1.1.3. The above lemma can be extended by iteration to the case of $n$ elements $x_{1}, \ldots, x_{n} \in A$ such that $x_{i} x_{j}=\lambda_{i j} x_{j} x_{i}$ for a multiplicatively skew-symmetric matrix $\left(\lambda_{i j}\right)_{1 \leq i, j \leq n}$ with entries in $\mathbb{k}^{\times}$such that $\lambda_{i i}=1$ for any $1 \leq i \leq n$.

Lemma 1.1.4. Let $B$ a $\mathbb{k}$-subalgebra of $A$ which is noetherian, and such that $A$ is finitely generated as left $B$-module. If the set $S$ of nonzero elements of $B$ is a left and right Ore subset in $A$, then the localized ring $A S^{-1}=S^{-1} A$ is equal to Frac $A$.

Proof. Let $a$ be a nonzero element of $A$. We consider the ascending chain $\left(M_{n}\right)_{n \geq 0}$ of left $B$-submodules of $A$ defined by $M_{n}=B+B a+B a^{2} \cdots+B a^{n}$ for any integer $n \geq 0$. The $B$-module $A$ is noetherian as a finitely generated module over a noetherian ring, and then $a^{p+1} \in M_{p}$ for some $p \geq 0$. There exist some elements $b_{0}, b_{1}, \ldots, b_{p} \in B$ such that $a^{p+1}+b_{p} a^{p}+\cdots+b_{1} a+b_{0}=0$. Choosing $p$ minimal we can suppose without restriction (up to simplifying by a power of $a$ in the domain $A)$ that $b_{0} \neq 0$. Then $\left(a^{p}+b_{p} a^{p-1}+\cdots+b_{1}\right) a=$ $-b_{0}$. It follows that the inverse in Frac $A$ of any nonzero element $a \in A$ can be written $a^{-1}=b_{0}^{-1} u$ for some $u \in A$ and $b_{0} \in S$, or equivalently $a^{-1}=v c_{0}^{-1}$ for some $v \in A$ and $c_{0} \in S$ because $S$ is a left and right Ore subset. We conclude that Frac $A=A S^{-1}=S^{-1} A$. 
1.2. The superalgebra structure on the skewfield of fractions of a superalgebra. We fix in this subsection a superalgebra $A$. Hence $A=$ $A_{0} \oplus A_{1}$ is a $\mathbb{Z}_{2}$-graded algebra, where $A_{0}$ is the subalgebra of even elements (which contains the base field $\mathbb{k}$ ), and $A_{1}$ is the $A_{0}$-module of odd elements.

Lemma 1.2.1. We suppose that $A=A_{0} \oplus A_{1}$ is a left and right Ore domain. Then denoting $S_{0}=A_{0} \backslash\{0\}$, the following conditions are satisfied:

(R) for any $a \in A$ and $s \in S_{0}$, there exist $b \in A$ and $t \in S_{0}$ such that $a t=s b$,

(L) for any $a \in A$ and $s \in S_{0}$, there exist $c \in A$ and $u \in S_{0}$ such that $u a=c s$.

In other words, $S_{0}$ is a right and left Ore set in $A$.

Proof. We prove first the result for homogeneous $a$. We fix $a_{i} \in A_{i}$ and

$s \in S_{0}$. By assumption there exist $b \in A$ and $t \in S$ such that $a_{i} t=s b$. We can decompose $b=b_{0}+b_{1}$ and $t=t_{0}+t_{1}$ with $b_{0}, t_{0} \in A_{0}$ and $b_{1}, t_{1} \in A_{1}$. Then $a_{i} t_{0}+a_{i} t_{1}=s b_{0}+s b_{1}$. For $i=0$, it follows that $a_{0} t_{0}=s b_{0}$ and $a_{0} t_{1}=s b_{1}$. If $t_{0} \neq 0$, the first equality gives the desired result. If $t_{0}=0$, then $t_{1} \neq 0$ and $a_{0} t_{1}^{2}=s b_{1} t_{1}$ gives the result. For $i=1$, we obtain $a_{1} t_{0}=b_{1}$ and $a_{1} t_{1}=s b_{0}$ and we conclude in the same way.

Now let $a$ be an element of $A$ and $s$ an element of $S_{0}$. We decompose $a=a_{0}+a_{1}$ with $a_{0} \in A_{0}$ and $a_{1} \in A_{1}$. Applying the first step of the proof to $a_{0}$ and $s$, there exist $c \in A_{0}, u \in S_{0}$ such that $a_{0} u=s c$. Similarly there exist $d \in A_{1}, v \in S_{0}$ such that $a_{1} v=s d$. Applying now the first step to $u, v \in S_{0}$, there exist $x \in S_{0}$ and $y \in A_{0}$ such that $u x=v y$. Since $A$ is a domain, we have more precisely $y \in S_{0}$. Denoting $t=u x=v y \in S_{0}$, we obtain $s c x=a_{0} u x=a_{0} t$ and $s d y=a_{1} v y=a_{1} t$. Finally the elements $b=c x+d y \in A$ and $t \in S_{0}$ satisfy $s b=s c x+s d y=\left(a_{0}+a_{1}\right) t=a t$. Hence property $(\mathrm{R})$ is satisfied. The proof of $(\mathrm{L})$ is similar.

Theorem 1.2.2. Let $A=A_{0} \oplus A_{1}$ a $\mathbb{k}$-superalgebra which is a noetherian domain. If $A$ is finitely generated as left $A_{0}$-module, then:

(i) Any element $f \in$ Frac $A$ can be written $f=a s^{-1}=t^{-1} b$ with $a, b \in A$ and $s, t \in A_{0} \backslash\{0\}$.

(ii) Frac $A=F_{0} \oplus F_{1}$ is a superalgebra, where $F_{0}$ (respectively $F_{1}$ ) is the subspace of fractions which can be written with homogeneous numerator and denominator of same parity (respectively opposite parity).

(iii) $A=A_{0} \oplus A_{1}$ is a subsuperalgebra of Frac $A=F_{0} \oplus F_{1}$.

(iv) $F_{0}$ is equal to the skewfield Frac $A_{0}$.

Proof. We denote by $\sigma$ the automorphism of the $\mathbb{k}$-algebra $A$ defined by $\sigma\left(a_{0}+a_{1}\right)=a_{0}-a_{1}$ for all $a_{0} \in A_{0}, a_{1} \in A_{1}$. Then $A_{0}$ is the subalgebra of invariants $A^{G}$ of the noetherian algebra $A$ under the action of the finite group $G$ generated by $\sigma$. Hence $A_{0}$ is noetherian ([21] corollary 1.12) and point (i) follows from direct application of lemmas 1.1.4 and 1.2.1

To prove that $F_{0}$ is a $\mathbb{k}$-subspace of Frac $A$, we consider $f=a s^{-1}$ and $g=b t^{-1}$ in $F_{0}$. We can suppose without lost of generality that $a, b \in A_{0}$ 
and $s, t \in S_{0}$. By lemma 1.2.1, there exist $c, d \in S_{0}$ such that $s c=t d$ and the element $e=s c=t d \in S_{0}$ satisfies $f=a c e^{-1}$ and $g=b d e^{-1}$. Hence $f+g=(a c+b d) e^{-1}$ lies in $F_{0}$. The proof is similar for $F_{1}$ and we have clearly Frac $A=F_{0} \oplus F_{1}$ using point (i) of the theorem. Finally, for any pair of elements $f=a s^{-1} \in F_{i}$ and $g=b t^{-1} \in F_{j}$ with $s, t \in S_{0}, a \in A_{i}$, $b \in A_{j}, i, j \in \mathbb{Z}_{2}$, we can introduce by lemma 1.2.1 some elements $c \in A_{j}$ and $u \in S_{0}$ such that $s c=b u$. Then $f g=a c(t u)^{-1}$ with $a c \in A_{i+j}$ and $s t \in S_{0}$. In other words $F_{i} F_{j} \subset F_{i+j}$ and the proof of (ii) is complete.

Assertions (iii) and (iv) are then clear.

We list here some classical situations where the previous theorem applies.

Example 1.2.3. Enveloping algebras of Lie superalgebras. We consider the case where the superalgebra $A$ is the enveloping algebra $\mathcal{U}(\mathfrak{g})$ of a finite dimensional complex Lie superalgebra $\mathfrak{g}=\mathfrak{g}_{0} \oplus \mathfrak{g}_{1}$. It follows from PBW theorem (see theorem 6.1.2 in [24]) that $\mathcal{U}(\mathfrak{g})$ is a finitely generated left module over its even part. The algebra $\mathcal{U}(\mathfrak{g})$ is always noetherian (see for instance [7] $\S 3$ proposition 1, or [24]). The question of being or not a domain is more delicate. If there exists in $\mathfrak{g}_{1}$ a nonzero element $x$ such that $[x, x]=0$, then $x$ is clearly nilpotent in $\mathcal{U}(\mathfrak{g})$ and $\mathcal{U}(\mathfrak{g})$ is not a domain. A nontrivial key result (see theorem 1 of [5], or theorem 17.1.1 in [24] following [8]) asserts that this condition is also sufficient: $\mathcal{U}(\mathfrak{g})$ is a domain if and only if $\mathfrak{g}$ doesn't contain any odd element $x$ such that $[x, x]=0$. Of all the cases of the classification of classical simple Lie superalgebras, the only one where the enveloping algebra is a domain is the orthosymplectic Lie superalgebra $\mathfrak{o s p}(1,2 n), n \geq 1$, see lemma 1 of [23], or pages 17-20 of [7]. We will come back in the next section to the case of $\mathfrak{o s p}(1,2)$, and in example 1.3 .2 to the cases of the enveloping algebras of some nilpotent or solvable Lie subsuperalgebras of $\mathfrak{o s p}(1,2 n)$.

Example 1.2.4. Iterated Ore extensions. Let $A$ be an iterated Ore extension in $n$ variables $x_{1}, \ldots, x_{n}$ (in this order) over $\mathbb{k}$. The family of monomials $\left(x_{1}^{j_{1}} x_{2}^{j_{2}} \cdots x_{n}^{j_{n}}\right)_{j \in \mathbb{N}^{n}}$ is a $\mathbb{k}$-basis of $A$. Suppose that $A$ is equipped with a structure of superalgebra such that $x_{p}, \ldots, x_{n}$ are odd for some $1 \leq p \leq n$ and $x_{i}$ even for $1 \leq i \leq p-1$ (if $p>1$ ). Then $A$ is generated by $1, x_{p}, \ldots, x_{n}$ as a left module over its even part $A_{0}$. Since $A$ is clearly a noetherian domain, theorem 1.2 .2 applies.

This generic situation covers many significant examples. The polynomial superalgebra $\widetilde{\mathcal{O}}\left(\mathbb{k}^{n}\right)$ is the superalgebra generated over $\mathbb{k}$ by $n$ odd generators $y_{1}, \ldots, y_{n}$ satisfying relations $y_{i} y_{j}=-y_{j} y_{i}$ for any $1 \leq i \neq j \leq n$. We have $\widetilde{\mathcal{O}}\left(\mathbb{k}^{n}\right) \simeq \mathcal{O}(\mathbb{k})^{\widehat{\otimes} n}$ where $\widehat{\otimes}$ denotes the tensor product in the category of superalgebras (see (1.1.5) in [10]).

More generally, the polynomial algebra $A=\mathbb{k}_{\Lambda}\left[x_{1}, \ldots, x_{n}\right]$ generated by $n$ variables $x_{1}, \ldots, x_{n}$ with relations $x_{i} x_{j}=\lambda_{i j} x_{j} x_{i}$ for all $1 \leq i, j \leq n$, for some multiplicatively skew-symmetric matrix $\Lambda=\left(\lambda_{i j}\right)_{1 \leq i, j \leq n}$ with entries in $\mathbb{k}^{\times}$satifying $\lambda_{i i}=1$ for any $1 \leq i \leq n$, can be equipped with a structure 
of superalgebra assigning parity 1 to some $x_{p}, \ldots, x_{n}$ and parity 0 to others. Theorem 1.2 .2 applies.

This is also the case for the Weyl superalgebra $\widetilde{A}_{n}(\mathbb{k})$, which is the superalgebra generated over $\mathbb{k}$ by $2 n$ odd generators $y_{1}, \ldots, y_{n}$ and $x_{1}, \ldots, x_{n}$ satisfying relations:

$$
x_{i} x_{j}+x_{j} x_{i}=y_{i} y_{j}+y_{j} y_{i}=x_{i} y_{j}+y_{j} x_{i}=0 \text { and } x_{i} y_{i}-y_{i} x_{i}=1
$$

for all $1 \leq i \neq j \leq n$. We have $\widetilde{A}_{n}(\mathbb{k}) \simeq \widetilde{A}_{1}(\mathbb{k})^{\widehat{\otimes} n}$.

1.3. Rational equivalence for superalgebras. The sufficient conditions of theorem 1.2.2 combined with the content of lemma 1.1 .2 and corollary 1.1 .3 naturally leads to the following definitions.

Definitions 1.3.1. Let $A=A_{0} \oplus A_{1}$ and $B=B_{0} \oplus B_{1}$ be two $\mathbb{k}$-superalgebras. We suppose that $A$ and $B$ are noetherian domains, and are finitely generated as left $A_{0}$-module and $B_{0}$-module respectively.

We say that the superalgebras $A$ and $B$ are s-rationally equivalent if the superalgebras Frac $A$ and Frac $B$ are isomorphic.

We say that the superalgebras $A$ and $B$ are strongly s-rationally equivalent if there exist homogeneous elements $x \in A$ and $y \in B$ generating a right and left Ore subset $X=\left\{x^{n}\right\}_{n \geq 0}$ in $A$ and a right and left Ore subset $Y=$ $\left\{y^{n}\right\}_{n \geq 0}$ in $B$ such that the superalgebras $A X^{-1}=B Y^{-1}$ are isomorphic.

It is clear that the strong s-rational equivalence implies the s-rational equivalence.

Remark 1.3.2. In order to illustrate the above notions, we end this section by revisiting the main theorem of a precedent paper 3] devoted to the enveloping algebras of the nilpotent positive subsuperalgebra $\mathfrak{n}^{+}$and the solvable Borel subsuperalgebra $\mathfrak{b}^{+}$of the orthosymplectic complex Lie superalgebra. It was proved that the skewfields of fractions of $\mathcal{U}\left(\mathfrak{n}^{+}\right)$and $\mathcal{U}\left(\mathfrak{b}^{+}\right)$are isomorphic as algebras to the skewfields of fractions of $A_{n(n-1) / 2}(\mathbb{C}) \otimes \widetilde{\mathcal{O}}\left(\mathbb{C}^{n}\right)$ and $A_{n(n-1) / 2}(\mathbb{C}) \otimes \widetilde{A}_{n}\left(\mathbb{C}^{n}\right)$ respectively.

The interested reader could verify that the proofs detailed in section 3 of [3] establish the existence of the desired isomorphisms at the level of localizations only by the multiplicative set generated by $n$ homogeneous elements $y_{1}, \ldots, y_{n}$ satisfying $y_{i} y_{j}= \pm y_{j} y_{i}$ (see [3] notations 3.2.2 and lemma 3.2 .3 , and then applying corollary 1.1 .3 by the multiplicative set generated by their product. Moreover the arguments explained in examples 1.2 .3 and 1.2.4 show that theorem 1.2 .2 applies to the superalgebras under consideration. Thus we can finally formulate on the basis of the same proof a reinforced version of the theorem in the category of superalgebras:

(1) the superalgebra $\mathcal{U}\left(\mathfrak{n}^{+}\right)$is strongly s-rationally equivalent to the superalgebra $A_{1}(\mathbb{C})^{\otimes n(n-1) / 2} \widehat{\otimes} \widetilde{\mathcal{O}}(\mathbb{C})^{\otimes} n$,

(2) the superalgebra $\mathcal{U}\left(\mathfrak{b}^{+}\right)$is strongly s-rationally equivalent to the superalgebra $A_{1}(\mathbb{C})^{\otimes n(n-1) / 2} \widehat{\otimes} \widetilde{A}_{1}(\mathbb{C})^{\widehat{\otimes} n}$, 
with the natural convention that $A_{1}(\mathbb{C})$ denotes the superalgebra defined on the Weyl algebra $A_{1}(\mathbb{C})$ assigning parity 0 to the generators.

\section{From enveloping algebra to Quantum EnVeloping Algebra of $\mathfrak{o s p}(1,2)$}

2.1. Rational equivalence for the classical enveloping algebra of $\mathfrak{o s p}(1,2)$. We return briefly here to the case of the classical enveloping algebra of the superalgebra $\mathfrak{o s p}(1,2)$. The goal is dual: to deepen in terms of strongly s-rational equivalence in the category of superalgebras the results of [2], and to introduce some parallel with the properties of the quantum analog studied in the next paragraph. We suppose in part 2.1 that $\mathbb{k}$ is of characteristic zero.

Definition 2.1.1. The orthosymplectic Lie superalgebra $\mathfrak{o s p}(1,2)$ is generated over $\mathbb{k}$ by $x, y, k, e, f$ with nonzero brackets:

$$
[k, x]=x, \quad[k, y]=-y, \quad[x, y]=2 k, \quad[x, x]=4 e, \quad[y, y]=-4 f,
$$

where the generators $x, y$ are odd and $k, e, f$ are even. This is a simple classical Lie superalgebra and one of the rare cases where the enveloping algebra is a domain, see example 1.2 .3 .

Notation 2.1.2. Let $\mathbf{S}_{3}(\mathbb{k})$ be the superalgebra generated over $\mathbb{k}$ by three generators $x, y, z$ satisfying:

$$
x y-y x=1, \quad x z=-z x, \quad y z=-z y,
$$

where $x$ and $y$ are odd, and $z$ is even. As an algebra, $\mathbf{S}_{3}(\mathbb{k})$ is the iterated Ore extension $\mathbb{k}[y]\left[x ; \partial_{y}\right][z ; \tau]$, where $\tau$ is the automorphism of $\mathbb{k}[y]\left[x ; \partial_{y}\right]$ defined by $\tau(x)=-x$ and $\tau(y)=-y$. Using lemma 1.1.1 we also consider the localization $\mathbf{S}_{3}^{\prime}=\mathbb{k}\left[y^{ \pm 1}\right]\left[x ; \partial_{y}\right][z ; \tau]$.

With the notations introduced in example 1.2.4, the superalgebras $\mathbf{S}_{3}(\mathbb{k})$ and $\widetilde{A}_{1}(\mathbb{k}) \widehat{\otimes} \widetilde{\mathcal{O}}(\mathbb{k})$ are isomorphic in the category of associative algebras. The difference is about the grading: $x$ and $y$ are odd in both superalgebras but $z$ is even in the first one and odd in the second one. We will see in the following theorem that they are not isomorphic as superalgebras nor even s-rationally equivalent.

\section{Theorem 2.1.3.}

(i) The superalgebra $\mathcal{U}(\mathfrak{o s p}(1,2))$ is strongly s-rationally equivalent to the superalgebra $\mathbf{S}_{3}(\mathbb{k})$.

(ii) The superalgebras $\mathcal{U}(\mathfrak{o s p}(1,2))$ and $\widetilde{A}_{1}(\mathbb{k}) \widehat{\otimes} \widetilde{\mathcal{O}}(\mathbb{k})$ are rationally equivalent in the category of associative algebras, but are not s-rationally equivalent.

Proof. Point (i) is a graded improvement of proposition 2.2 in [2]. The algebra $U:=\mathcal{U}(\mathfrak{o s p}(1,2))$ is generated by $x, y, k$ with relations:

$$
k x-x k=x, \quad k y-y k=-y, \quad y x=-x y+2 k,
$$


and the superalgebra structure is defined assigning parity zero to $k$ and parity 1 to $x$ and $y$. It is clearly an iterated Ore extension $U=\mathbb{k}[x][k ; \delta][y ; \tau, d]$, where $\delta$ is the derivation $x \partial_{x}$ in $\mathbb{k}[x], \tau$ is the automorphism of $\mathbb{k}[x][k ; \delta]$ defined by $\tau(x)=-x$ and $\tau(k)=k+1$, and $d$ is the $\tau$-derivation of $\mathbb{k}[x][k ; \delta]$ defined by $d(x)=2 k$ and $d(k)=0$. By lemma 1.1.1, we can extend canonically $\delta, \tau$ and $d$ to the Laurent polynomial algebra $\mathbb{k}\left[x^{ \pm 1}\right]$ and consider the localization $U^{\prime}:=\mathbb{k}\left[x^{ \pm 1}\right][k ; \delta][y ; \tau, d]$ of $U$. The even element $z:=x y-y x+1=2 x y-2 k+1$ satisfies $z x=-x z$ and $z k=k z$. Since $y=\frac{1}{2}(x)^{-1}(z+2 k-1)$ in $U^{\prime}$, we have $U^{\prime}=\mathbb{k}\left[x^{ \pm 1}\right][k ; \delta]\left[z ; \tau^{\prime}\right]$ with $k x-x k=x, z k=k z$ and $z x=-x z$. We introduce the odd element $h:=x^{-1} k$ and obtain $U^{\prime}=\mathbb{k}\left[x^{ \pm 1}\right]\left[h ; \partial_{x}\right]\left[z ; \tau^{\prime}\right]$ with $h x-x h=1, z x=-x z$ and $z h=-h z$. Hence the superalgebras $U^{\prime}$ and $\mathbf{S}_{3}^{\prime}(\mathbb{k})$ are isomorphic and point (i) is proved.

We consider now the separation result (ii). By point (i) we have Frac $U=$ Frac $\mathbf{S}_{3}(\mathbb{k})$ as superalgebras. This skewfield $F$ can be described as an iterated skewfield of rational functions $F=\mathbb{k}(z)(y ; \tau)(x ; \tau, d)$ where the automorphism $\tau$ is defined by $\tau(z)=-z$ and $\tau(y)=y$ and the $\tau$-derivation $d$ is defined by $d(z)=0$ and $d(y)=1$. Using the classical embedding of $F$ into the Laurent series skewfield $\mathbb{k}(z)(y ; \tau)\left(\left(x^{-1} ; \tau^{-1},-d \tau^{-1}\right)\right)$, see [12] paragraph 1.4 or [16] exercise 1ZB, we can prove by direct calculations that an element $f \in F$ satisfying $y f=-f y$ necessarily satisfies $f \in \mathbb{k}(z)(y ; \sigma)$. Similarly $x f=-f x$ implies $f \in \mathbb{k}(z)(x ; \sigma)$. Consider now the superalgebra structure on Frac $\mathbf{S}_{3}(\mathbb{k})$ extending that of $\mathbf{S}_{3}(\mathbb{k})$ (see theorem 1.2.2). Assume that there exists in $\operatorname{Frac}\left(\mathbf{S}_{3}(\mathbb{k})\right)$ an odd element $f$ which is supercentral; that means that $f g=-g f$ for any odd element $g$ in Frac $\left(\mathbf{S}_{3}(\mathbb{k})\right)$ and $f h=h f$ for any even element $h$ in Frac $\left(\mathbf{S}_{3}(\mathbb{k})\right)$. In particular, $f x=-x f$ and $f y=-y f$ which implies $f \in \mathbb{k}(z)$ by the previous calculation. This is impossible because all elements of $\mathbb{k}(z)$ are even in $\operatorname{Frac}\left(\mathbf{S}_{3}(\mathbb{k})\right)$. On the contrary the superalgebra $\widetilde{A}_{1}(\mathbb{k}) \widehat{\otimes} \widetilde{\mathcal{O}}(\mathbb{k})$ contains supercentral odd elements, for instance the generator $z$ of $\widetilde{\mathcal{O}}(\mathbb{k})=\mathbb{k}[z]$ in the presentation $(2)$. Then the algebras $\mathbf{S}_{3}(\mathbb{k})$ and $\widetilde{A}_{1}(\mathbb{k}) \widehat{\otimes} \widetilde{\mathcal{O}}(\mathbb{k})$ are isomorphic as algebras but their skewfield of fractions are not isomorphic as superalgebras.

Remark 2.1.4. Point (ii) of the theorem shows that, while tensor products of Weyl superalgebras and polynomial superalgebras were sufficient to describe the superalgebra structure on the skewfield of fractions in the nilpotent or solvable situations of the examples in 1.3 .2 , this is no longer the case for the simple classical Lie superalgebra $\mathfrak{o s p}(1,2)$.

Remarks 2.1.5 (embedding problems). (i) There exists a canonical embedding of $\mathcal{U}(\mathfrak{s l}(2))$ into $\mathcal{U}(\mathfrak{o s p}(1,2))$. More precisely, the even part of $\mathfrak{o s p}(1,2)$ being the Lie algebra $\mathfrak{s l}(2)$, the enveloping algebra $V:=\mathcal{U}(\mathfrak{s l}(2))$ embeds into the even part $U_{0}$ of $\mathcal{U}\left(\mathfrak{o s p}(1,2)\right.$. Explicitly, the even elements $e:=\frac{1}{2} x^{2}$, $f:=-\frac{1}{2} y^{2}$ and $k$ satisfy $[k, e]=2 e,[k, f]=-2 f$ and $[e, f]=k$, and generate a copy of $V$ in $U_{0}$. The inclusion $V \subset U_{0}$ is strict: $x y \in U_{0}$ and $x y \notin V$. 
(ii) Conversely, there is no embedding of $\mathcal{U}(\mathfrak{o s p}(1,2))$ into $\mathcal{U}(\mathfrak{s l}(2))$. This follows from the fact that $\mathcal{U}(\mathfrak{o s p}(2))$ contains two elements $x$ and $z$ such that $x z=-z x$; by theorem 3.10.(a) of [1] using the rational invariant $G$ introduced in definition 3.8 of [1, this is impossible in $\mathcal{U}(\mathfrak{s l}(2))$ because its skewfield of fractions is a Weyl skewfield $D_{1}$ over the center $\mathbb{k}(\omega)$, with $\omega$ the Casimir operator.

2.2. Quantum polynomial superalgebra. We will use in all forthcoming results the following particular case of the superalgebras $\mathbb{k}_{\Lambda}\left[x_{1}, \ldots, x_{n}\right]$ previously mentionned in 1.2 .4

Definition 2.2.1. Let $q$ be an element of $\mathbb{k}^{\times}, q \neq \pm 1$. A $q$-polynomial superalgebra is a polynomial superalgebra in $n$ variables $x_{1}, \ldots, x_{n}$ where the $x_{j}$ 's are homogeneous (even or odd) and satisfy for all $1 \leq i, j \leq n$ commutation relations $x_{i} x_{j}=x_{j} x_{i}$, anticommutation relations $x_{j} x_{j}=-x_{j} x_{i}$, or quantum commutation relations $x_{j} x_{j}=q^{\alpha_{i j}} x_{j} x_{i}, \alpha_{i j} \in \mathbb{Z}, \alpha_{i j} \neq 0$.

Notation 2.2.2. In order to have a synthetic view of these polynomial algebras, we use a graphical representation by diagrams. The vertices refer to the generators, with a white circle for an even generator and a gray circle for an odd one. The edges refer to the relations between the generators they connect: an oriented $q^{\alpha}$-weighted solid edge for a $q^{\alpha}$-commutation, a dotted edge for an anticommutation, and no edge for a commutation. The first examples appear in 2.3 .3 or 3.1 .2 below.

2.3. Quantum enveloping algebra of $\mathfrak{o s p}(1,2)$. Just as there is a wellknown quantum version $\mathcal{U}_{q}(\mathfrak{s l}(2))$ of the enveloping algebra $\mathcal{U}(\mathfrak{s l}(2))$ of the Lie algebra $\mathfrak{s l}(2)$, many authors have considered in the literature a superalgebra $\mathcal{U}_{q}(\mathfrak{o s p}(1,2))$ as a quantization of the enveloping algebra $\mathcal{U}(\mathfrak{o s p}(1,2))$ considered in 2.1. This give rise to the following general picture:

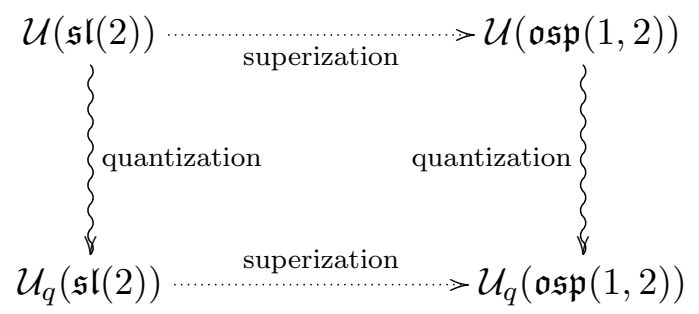

Definition 2.3.1 ([4, 22, 26, 28]). Let $q$ be an element of $\mathbb{k}^{\times}, q \neq \pm 1$. The quantum enveloping algebra of the Lie superalgebra osp $(1,2)$ is the superalgebra $\mathcal{U}_{q}(\mathfrak{o s p}(1,2))$ generated over $\mathbb{k}$ by $e, f, k^{ \pm 1}$ with relations:

$$
k k^{-1}=k^{-1} k=1, \quad k e=q e k, \quad k f=q^{-1} f k, \quad \text { ef }+f e=\frac{k-k^{-1}}{q-q^{-1}}
$$

and $\mathbb{Z}_{2}$-grading such that $e$ and $f$ are odd, and $k^{ \pm 1}$ is even.

In view of a quantum analogue of proposition 2.1.3, we introduce the following multiplicative version of the algebra defined in 2.1 .2 
Notation 2.3.2. For any element $q \in \mathbb{k}^{\times}, q \neq \pm 1$, we denote by $\mathbf{S}_{3}^{q}(\mathbb{k})$ the $q$-polynomial superalgebra generated over $\mathbb{k}$ by three generators $x, y, z$ satisfying:

$$
x y=q y x, x z=-z x, y z=-z y, x \text { and } y \text { are odd, } z \text { is even. }
$$

It can be seen as an iterated Ore extension $\mathbb{k}[y][x ; \sigma][z ; \tau]$, where $\sigma$ is the automorphism of $\mathbb{k}[y]$ defined by $\sigma(y)=q y$ and $\tau$ is the automorphism of $\mathbb{k}[y][x ; \sigma]$ defined by $\tau(x)=-x$ and $\tau(y)=-y$. By lemma 1.1.1, we also consider the localization $\mathbf{S}_{3}^{\prime \prime}{ }_{3}=\mathbb{k}\left[y^{ \pm 1}\right]\left[x^{ \pm 1} ; \sigma\right][z ; \tau]$.

Proposition 2.3.3. The superalgebra $\mathcal{U}_{q}(\mathfrak{o s p}(1,2))$ is strongly s-rationally equivalent to the q-polynomial superalgebra $\mathbf{S}_{3}^{q}(\mathbb{k})$, represented by the diagram:

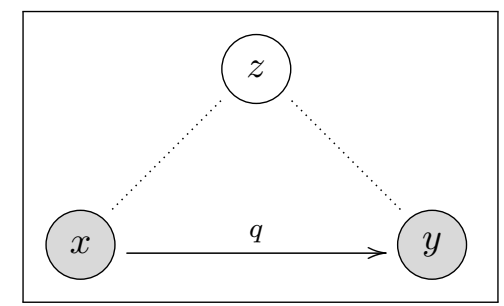

Proof. We denote $U_{q}:=\mathcal{U}_{q}(\mathfrak{o s p}(1,2))$. It follows from the definition that

$$
U_{q}=\mathbb{k}\left[k^{ \pm 1}\right][e ; \sigma][f ; \tau, \delta],
$$

with a PBW basis $\mathcal{B}=\left(k^{a} e^{b} f^{c}\right)_{a \in \mathbb{Z}, b, c \in \mathbb{N}}$ (see [4] proposition 2), where $\sigma$ is the automorphism of $\mathbb{k}\left[k^{ \pm 1}\right]$ defined by $\sigma(k)=q^{-1} k, \tau$ is the automorphism of $\mathbb{k}\left[k^{ \pm 1}\right][e ; \sigma]$ defined by $\tau(k)=q k$ and $\tau(e)=-e$, and $\delta$ is the $\tau$-derivation of $\mathbb{k}\left[k^{ \pm 1}\right][e ; \sigma]$ defined by $\delta(k)=0$ and $\delta(e)=\frac{1}{q-q^{-1}}\left(k-k^{-1}\right)$. We are in the context of example 1.2.4. Following [4], we introduce the super Casimir element, that is the even element:

$$
\begin{aligned}
s & :=q^{-1 / 2} k-q^{1 / 2} k^{-1}-\left(q^{1 / 2}+q^{-1 / 2}\right)\left(q-q^{-1}\right) e f \\
& =-q^{1 / 2} k+q^{-1 / 2} k^{-1}+\left(q^{1 / 2}+q^{-1 / 2}\right)\left(q-q^{-1}\right) f e .
\end{aligned}
$$

By elementary calculations using relations (6) we show that:

$$
s k^{ \pm 1}=k^{ \pm 1} s, \text { se }=-e s, s f=-f s .
$$

Then $s$ commutes with any even element and anticommutes with any odd element. In particular $s^{2}$ lies in the center of $U_{q}$. Using lemma 1.1.1, we consider the localization $U_{q}^{\prime}=\mathbb{k}\left[k^{ \pm 1}\right]\left[e^{ \pm 1} ; \sigma\right][f ; \tau, \delta]$ of $U_{q}$ with respect to the powers of the odd generator $e$. By (7), we have in $U_{q}^{\prime}$ :

$$
f=-\frac{1}{\left(q^{1 / 2}+q^{-1 / 2}\right)\left(q-q^{-1}\right)} e^{-1} s+e^{-1} \frac{1}{\left(q^{1 / 2}+q^{-1 / 2}\right)\left(q-q^{-1}\right)}\left[q^{-1 / 2} k-q^{1 / 2} k^{-1}\right] .
$$

Then, by change of generator, $U_{q}^{\prime}$ appears as the iterated Laurent extension:

$$
U_{q}^{\prime}=\mathbb{k}\left[k^{ \pm 1}\right]\left[e^{ \pm 1} ; \sigma\right][s ; \gamma],
$$

where the $\mathbb{k}$-automorphism $\gamma$ is the involution $k \mapsto k$ and $e \mapsto-e$ corresponding to the relations: $k e=q e k, s e=-e s$ and $s k=k s$. In the 
expression (9) of $U_{q}^{\prime}$, we can replace the even generator $k$ by the odd generator $k_{0}:=e^{-1} k$. The relations become $k_{0} e=q e k_{0}, s e=-e s, s k_{0}=-s k_{0}$. We conclude that the superalgebra $U_{q}^{\prime}=\mathbb{k}\left[k_{0}^{ \pm 1}\right]\left[e^{ \pm 1} ; \sigma\right][s ; \gamma]$ is isomorphic to the superalgebra $\mathbf{S}_{3}^{\prime \prime}(\mathbb{k})$ introduced in 2.3 .2 .

We obtain as an immediate consequence the following description of the center of $\mathcal{U}_{q}(\mathfrak{o s p}(1,2))$, already proved in [4] proposition 3 , and of the center of its superskewfield of fractions.

Corollary 2.3.4. We suppose that $q$ is not a root of one and denote by $s$ the super Casimir element defined by (7). The center of $\mathcal{U}_{q}(\mathfrak{o s p}(1,2))$ is the polynomial algebra $\mathbb{k}\left[s^{2}\right]$. The center of $\operatorname{Frac}\left(\mathcal{U}_{q}(\mathfrak{o s p}(1,2))\right)$ is the purely transcendental extension $\mathbb{k}\left(s^{2}\right)$.

Proof. With the notations used in the proof of the proposition, the skewfield of fractions $F:=\operatorname{Frac} U_{q}=\operatorname{Frac} U_{q}^{\prime}$ is the skewfield of rational functions $F=\mathbb{k}(s)\left(k_{0} ; \gamma\right)(e ; \sigma)$ where the automorphisms $\gamma$ and $\sigma$ are defined by $\gamma: s \mapsto-s$ and $\sigma: s \mapsto-s, k_{0} \mapsto q^{-1} k_{0}$. By classical methods of embeddings in skewfields of Laurent series (see for instance [1] section 1.1, or [16] exercise 1ZA) and because $q$ is not a root of one, we easily see that an element $f \in F$ commutes with $k_{0}$ and $e$ if and only if $f \in \mathbb{k}\left(s^{2}\right)$. It follows that the center of $F$ and the center of $\operatorname{Frac}\left(U_{q}\right)$ are equal to $\mathbb{k}\left(s^{2}\right)$. Since $s$ is an element of $U_{q}$, we deduce that the center of $U_{q}$ is $\mathbb{k}\left[s^{2}\right]$.

We have seen in 2.1.5 that in the classical case the enveloping algebra $\mathcal{U}(\mathfrak{s l}(2))$ appears as a subalgebra of the even part of the enveloping algebra $\mathcal{U}(\mathfrak{o s p}(1,2))$. A similar question can be asked for $\mathcal{U}_{q}(\mathfrak{o s p}(1,2))$ and the quantum algebra $\mathcal{U}_{q}(\mathfrak{s l}(2))$.

Theorem 2.3.5. The subalgebra generated in $\mathcal{U}_{q}(\mathfrak{o s p}(1,2))$ by the elements:

$$
X:=\left(q^{-1} k+q k^{-1}\right) e+\left(q^{-1}+2+q\right) e^{2} f, \quad Y:=-f, \quad K:=k^{2}
$$

is isomorphic to $\mathcal{U}_{q}(\mathfrak{s l}(2))$.

Proof. First step. It is clear by (5) that $K X=q^{2} X K$ and $K Y=q^{-2} Y K$. We introduce the notations $\lambda:=q^{-1}+2+q \in \mathbb{k}^{\times}$and $c:=q^{-1} k+q k^{-1} \in$ $\mathbb{k}\left[k^{ \pm 1}\right]$, so that $X=c e+\lambda e^{2} f$. Then the products $X Y=-c e f-\lambda e^{2} f^{2}$ and $Y X=-\sigma^{-1}(c) f e-\lambda(f e)(e f)$ appear as elements of the even part of $U_{q}$, which is the $\mathbb{k}$-subalgebra of $U_{q}$ generated by $k^{ \pm 1}, e^{2}, f^{2}, e f$. Some preliminary calculations in this even part involving the semi-Casimir element are necessary.

Second step: calculations in $\mathbb{k}\left[k^{ \pm 1}\right][s]$. We introduce the scalar:

$$
\hat{q}=\frac{1}{\left(q^{1 / 2}+q^{-1 / 2}\right)\left(q-q^{-1}\right)}
$$

and the following elements in $\mathbb{k}\left[k^{ \pm}\right]$:

$$
k^{\prime}=q^{-1 / 2} k-q^{1 / 2} k^{-1}, \quad k^{\prime \prime}=q^{1 / 2} k-q^{-1 / 2} k^{-1} .
$$


Then identities (7) and (8) can be rewritten:

$$
e f=-\hat{q} s+\hat{q} k^{\prime} \quad \text { and } \quad f e=\hat{q} s+\hat{q} k^{\prime \prime} .
$$

Recalling that $\sigma$ and $\tau$ are the automorphisms of $\mathbb{k}\left[k^{ \pm 1}\right]$ defined by $\sigma(k)=$ $q^{-1} k$ and $\tau(k)=q k$, see (6), we compute:

$$
\begin{aligned}
& e^{2} f^{2}=-\hat{q}^{2} s^{2}+\hat{q}^{2}\left(k^{\prime}-\sigma\left(k^{\prime}\right)\right) s+\hat{q}^{2} k^{\prime} \sigma\left(k^{\prime}\right), \\
& f^{2} e^{2}=-\hat{q}^{2} s^{2}+\hat{q}^{2}\left(\tau\left(k^{\prime \prime}\right)-k^{\prime \prime}\right) s+\hat{q}^{2} k^{\prime \prime} \tau\left(k^{\prime \prime}\right) .
\end{aligned}
$$

Third step: expression of the commutator $X Y-Y X$. We can now resume the calculation of $X Y$ and $Y X$ using identities (13) and (14):

$$
\begin{aligned}
-X Y & =c e f+\lambda e^{2} f^{2} \\
& =-\lambda \hat{q}^{2} s^{2}+\left[-c \hat{q}+\lambda \hat{q}^{2}\left(k^{\prime}-\sigma\left(k^{\prime}\right)\right] s+\hat{q} c k^{\prime}+\lambda \hat{q}^{2} k^{\prime} \sigma\left(k^{\prime}\right),\right. \\
-Y X & =\sigma^{-1}(c) f e+\lambda(f e)(e f) \\
& =\sigma^{-1}(c)\left(\hat{q} s+\hat{q} k^{\prime \prime}\right)+\lambda\left(\hat{q} s+\hat{q} k^{\prime \prime}\right)\left(-\hat{q} s+\hat{q} k^{\prime}\right) \\
& =-\lambda \hat{q}^{2} s^{2}+\left[\sigma^{-1}(c) \hat{q}+\lambda \hat{q}^{2}\left(k^{\prime}-k^{\prime \prime}\right)\right] s+\sigma^{-1}(c) \hat{q} k^{\prime \prime}+\lambda \hat{q}^{2} k^{\prime} k^{\prime \prime} .
\end{aligned}
$$

Then the difference $X Y-Y X$ is of degree $\leq 1$ in $\mathbb{k}\left[k^{ \pm 1}\right][s]$ with leading coefficient $u_{1}:=\hat{q}\left[c+\sigma^{-1}(c)+\lambda \hat{q}\left(\sigma\left(k^{\prime}\right)-k^{\prime \prime}\right)\right]$. On one hand:

$$
c+\sigma^{-1}(c)=\left(1+q^{-1}\right) k+(1+q) k^{-1} \text {. }
$$

On the other hand by (11):

$$
\begin{aligned}
\sigma\left(k^{\prime}\right)-k^{\prime \prime} & =\left(q^{-3 / 2} k-q^{3 / 2} k^{-1}\right)-\left(q^{1 / 2} k-q^{-1 / 2} k^{-1}\right) \\
& =\left(q^{-3 / 2}-q^{1 / 2}\right) k+\left(q^{-1 / 2}-q^{3 / 2}\right) k^{-1} \\
& =\left(q^{-1 / 2}-q^{1 / 2}\right)\left[\left(1+q^{-1}\right) k+(1+q) k^{-1}\right] .
\end{aligned}
$$

Then $u_{1}=\hat{q}\left[1+\lambda \hat{q}\left(q^{-1 / 2}-q^{1 / 2}\right)\right]\left[\left(1+q^{-1}\right) k+(1+q) k^{-1}\right]$. An auxiliary calculation using (10) gives:

$$
\lambda \hat{q}\left(q^{-1 / 2}-q^{1 / 2}\right)=\frac{\lambda\left(q^{-1 / 2}-q^{1 / 2}\right)}{\left(q^{1 / 2}+q^{-1 / 2}\right)\left(q-q^{-1}\right)}=\frac{-\lambda}{\left(q^{1 / 2}+q^{-1 / 2}\right)^{2}}=-1,
$$

and we conclude that $u_{1}=0$. Therefore

$$
\left.X Y-Y X=\hat{q}\left(\sigma^{-1}(c) k^{\prime \prime}-c k^{\prime}\right)\right)-\lambda \hat{q}^{2} k^{\prime}\left(\sigma\left(k^{\prime}\right)-k^{\prime \prime}\right) .
$$

It follows from the above calculations that $\lambda \hat{q}\left(\sigma\left(k^{\prime}\right)-k^{\prime \prime}\right)=-c-\sigma^{-1}(c)$, then:

$$
\begin{aligned}
X Y-Y X & =\hat{q}\left(\sigma^{-1}(c) k^{\prime \prime}-c k^{\prime}\right)-\hat{q} k^{\prime}\left(-c-\sigma^{-1}(c)\right) \\
& =\hat{q} \sigma^{-1}(c)\left(k^{\prime \prime}+k^{\prime}\right) \\
& =\hat{q}\left(k+k^{-1}\right)\left(q^{-1 / 2}+q^{1 / 2}\right)\left(k-k^{-1}\right) \\
& =\frac{k^{2}-k^{-2}}{q-q^{-1}}
\end{aligned}
$$


Fourth step: conclusion. It is clear by the definition of $X, Y, K$ that the monomials $\left(K^{p} Y^{m} X^{n}\right)_{p \in \mathbb{Z}, m, n \in \mathbb{N}}$ are linearly independent in the iterated Ore extension (6). They satisfy the relations $K X=q^{2} X K, K Y=q^{-2} Y K$ and $X Y-Y X=\frac{K-K^{-1}}{q-q^{-1}}$. By definition of $\mathcal{U}_{q}(\mathfrak{s l}(2))$ (see for instance [19] VI.1.1 or [9] 1.3.1), the proof is complete.

Remarks 2.3.6. (i) The following question remains still open to the best of our knowledge: do we have an embedding of the quantum algebra $\mathcal{U}_{q}(\mathfrak{s l}(2))$ in the even part of the superalgebra $\mathcal{U}_{q}(\mathfrak{o s p}(1,2))$ ?

(ii) In view of remark 2.1.5 and proposition 2.3.5, the question arises naturally of the possible embeddings between the four algebras considered in (4). The following proposition answers the question at the higher level of their skewfields of fractions.

Proposition 2.3.7. We suppose that $\mathbb{k}$ is of characteritic zero and that $q$ is not a root of one in $\mathbb{k}^{\times}$. Then we have the following possible and impossible algebra embeddings between the skewfields of fractions $\mathcal{K}(\mathfrak{s l}(2)), \mathcal{K}(\mathfrak{o s p}(1,2))$ $\mathcal{K}_{q}(\mathfrak{s l}(2))$ and $\mathcal{K}_{q}(\mathfrak{o s p}(1,2))$ of the four algebras considered in (4):

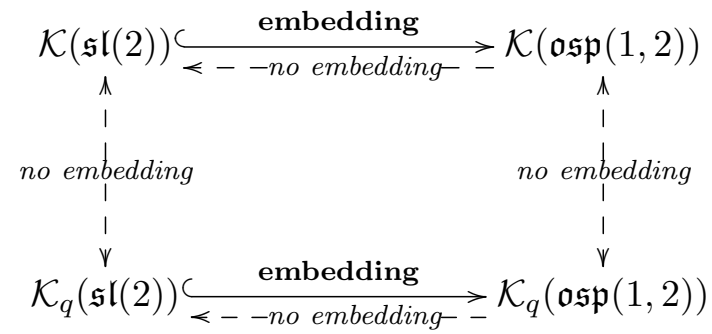

Proof. The canonical embedding of $\mathcal{U}(\mathfrak{s l}(2))$ into $\mathcal{U}(\mathfrak{o s p}(1,2))$ trivially induces an embedding of $\mathcal{K}(\mathfrak{s l}(2))$ into $\mathcal{K}(\mathfrak{o s p}(1,2))$. Conversely, we have already seen in remark 2.1.5.(ii) that $\mathcal{K}(\mathfrak{s l}(2))$ cannot contain a copy of $\mathcal{U}(\mathfrak{o s p}(1,2))$ and then a fortiori a copy of $\mathcal{K}(\mathfrak{o s p}(1,2))$. In the same way, proposition 2.3.5 implies the existence of a canonical embedding of $\mathcal{K}_{q}(\mathfrak{s l}(2))$ into $\mathcal{K}_{q}(\mathfrak{o s p}(1,2))$. By [1] proposition $4.3, \mathcal{K}_{q}(\mathfrak{s l}(2))$ is a quantum Weyl skewfield; then by [1] theorem $3.10 . \mathrm{b}$, it cannot contain pairs of elements $a, b$ satisfying $a b=-b a$ when $q$ is not a root of one. We deduce that there is no embedding of $\mathcal{K}_{q}(\mathfrak{o s p}(1,2))$ in $\mathcal{K}_{q}(\mathfrak{s l}(2))$.

Similarly, using proposition 3.9 and theorem 3.10 of [1, the two upward arrows may be justified by the fact that there are no pairs of elements $a, b$ satisfying $a b=q^{n} b a$ for $n \neq 0$ in $\mathcal{K}(\mathfrak{s l}(2))$ and $\mathcal{K}(\mathfrak{o s p}(1,2))$, and the two downward arrows by the fact that there are no pairs of elements $a, b$ satisfying $a b-b a=1$ in $\mathcal{K}_{q}(\mathfrak{s l}(2))$ and $\mathcal{K}_{q}(\mathfrak{o s p}(1,2))$.

\section{Some Subsuperalgebras of THE QUANTUM ENVELOPING ALGEBRA OF $\mathfrak{o s p}(1,4)$}

We fix an element $q \in \mathbb{k}^{\times}, q \neq \pm 1$. 
3.1. Quantum enveloping algebra of the nilpotent positive part of $\mathfrak{o s p}(1,4)$. For any $n \geq 2$, the superalgebra $\mathcal{U}_{q}\left(\mathfrak{o s p}^{+}(1,2 n)\right)$ is defined as the subsuperalgebra of $\mathcal{U}_{q}(\mathfrak{o s p}(1,2 n))$ generated by $n-1$ even generators $e_{1}, \ldots, e_{n-1}$ and one odd generator $e_{n}$ satisfying the quantum super Serre relations: see for instance [26] relations (9)-(12), 27] relations (3.2), 28] relations (3)-(4). Taking $q=1$ in the quantum super Serre relations gives exactly the super Serre relations (see $(2.30),(2.31),(2.32)$ in [1]). We study here the case $n=2$ denoting $\mathfrak{n}=\mathfrak{o s p} \mathfrak{p}^{+}(1,4)$.

Definition 3.1.1. The quantum enveloping algebra of the positive nilpotent part $\mathfrak{n}$ of the Lie superalgebra $\mathfrak{o s p}(1,4)$ is the superalgebra $\mathcal{U}_{q}(\mathfrak{n})$ generated over $\mathbb{k}$ by two elements $e_{1}, e_{2}$ satisfying the Serre relations:

$$
\begin{aligned}
& e_{1}^{2} e_{2}-\left(q+q^{-1}\right) e_{1} e_{2} e_{1}+e_{2} e_{1}^{2}=0, \\
& e_{2}^{3} e_{1}+\left(1-q-q^{-1}\right)\left(e_{2}^{2} e_{1} e_{2}+e_{2} e_{1} e_{2}^{2}\right)+e_{1} e_{2}^{3}=0,
\end{aligned}
$$

and $\mathbb{Z}_{2}$-grading such that $e_{1}$ is even and $e_{2}$ is odd.

Proposition 3.1.2. The superalgebra $\mathcal{U}_{q}(\mathfrak{n})$ is strongly s-rationally equivalent to the q-polynomial superalgebra $\mathbf{S}_{3}^{q}(\mathbb{k}) \widehat{\otimes} \mathcal{O}(\mathbb{k})$, represented by the diagram:

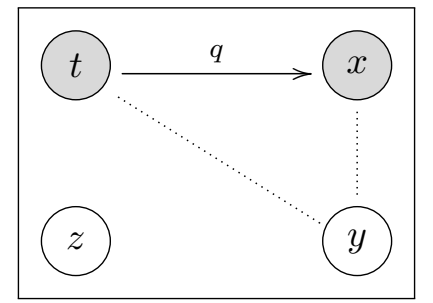

Proof. We denote $N_{q}:=\mathcal{U}_{q}(\mathfrak{n})$. A natural way to transform relation (16) is to introduce the odd element:

$$
x:=e_{1} e_{2}-q^{-1} e_{2} e_{1},
$$

so that (16) becomes simply $e_{1} x-q x e_{1}=0$. Then we consider relation (17) under the form $e_{2} y+y e_{2}=0$ where $y$ is even defined by:

$$
y:=x e_{2}-q e_{2} x=e_{2}^{2} e_{1}-\left(q+q^{-1}\right) e_{2} e_{1} e_{2}+e_{1} e_{2}^{2} .
$$

Straightforward calculations show that $e_{1} y=y e_{1}$ and $y x=-x y$. Hence we obtain a description of $N_{q}$ as an iterated Ore extension

$$
N_{q}=\mathbb{k}\left[e_{1}, y\right][x ; \sigma]\left[e_{2} ; \tau, \delta\right],
$$

with PBW basis $\mathcal{B}=\left(e_{1}^{a} y^{b} x^{c} e_{2}^{d}\right)_{a, b, c, d \in \mathbb{N}}$, where $\sigma, \tau$ and $\delta$ correspond to the commutation relations:

$$
\begin{array}{ll}
e_{1} y=y e_{1}, & x e_{1}=q^{-1} e_{1} x, \quad e_{1} e_{2}-q^{-1} e_{2} e_{1}=x, \\
x y=-y x, & e_{2} y=-y e_{2}, \quad x e_{2}-q e_{2} x=y .
\end{array}
$$


In this description $e_{1}$ and $y$ are even, $x$ and $e_{2}$ are odd. In particular we are in the context of example 1.2.4. Observe that it follows from (21) and (20) that $y^{2}$ lies in the center $Z\left(N_{q}\right)$ of $N_{q}$.

Another way to transform relation (17) is to introduce the even element

$$
u:=x e_{2}+e_{2} x=-q^{-1} y+\left(1+q^{-1}\right) x e_{2}
$$

in order to rewrite (17) under the form: $e_{2} u=q^{-1} u e_{2}$. We check that $u x=q^{-1} x u=$ and $u e_{1}=e_{1} u-(1+q) x^{2}$ and straightforward calculations show that the even element

$$
z:=e_{1} u-q^{2} u e_{1}
$$

commutes with $e_{1}, e_{2}, x$ and $y$. Then $z \in Z\left(N_{q}\right)$. We have the following explicit developments:

$$
\begin{aligned}
z & =\left(1-q^{2}\right) e_{1} u+q^{2}(1+q) x^{2} \\
& =\left(1-q^{2}\right)\left(1+q^{-1}\right) e_{1} x e_{2}+\left(q-q^{-1}\right) e_{1} y+q^{2}(1+q) x^{2}
\end{aligned}
$$

We denote by $N_{q}^{\prime}$ the localization of $N_{q}$ with respect to the multiplicative set generated by the homogeneous generators $e_{1}$ and $x$. By (25), we can replace in $N_{q}^{\prime}$ the generator $e_{2}$ by the central generator $z$, hence:

$$
N_{q}^{\prime}=\mathbb{k}\left[y, e_{1}^{ \pm}\right]\left[x^{ \pm} ; \sigma\right]\left[e_{2} ; \tau, \delta\right]=\mathbb{k}\left[y, e_{1}^{ \pm}\right]\left[x^{ \pm} ; \sigma\right][z] .
$$

Finally we replace the even generator $e_{1}$ by the odd one:

$$
t:=x^{-1} e_{1}=q e_{1} x^{-1}
$$

to obtain:

$$
N_{q}^{\prime}=\mathbb{k}[z]\left[t^{ \pm}\right]\left[x^{ \pm} ; \sigma\right][y ; \gamma] .
$$

By construction $y$ and $z$ are even, $x$ and $t$ are odd, $z$ is central, $t x=q x t$ and $y$ satisfies $y x+x y=y t+t y=0$. With the notations of 2.3 .2 and denoting $\mathcal{O}(\mathbb{k})=\mathbb{k}[z]$ for even $z$, the superalgebras $N_{q}^{\prime}$ and $\mathbf{S}_{3}^{\prime \prime}{ }_{3}^{q}(\mathbb{k}) \widehat{\otimes \mathcal{O}}(\mathbb{k})$ are isomorphic. Since the localizations are with respect to the powers of homogeneous $q$-commuting elements, the result follows from direct application of lemma 1.1 .2

Corollary 3.1.3. We suppose that $q$ is not a root of one and denote by $y$ and $z$ the generators defined by $(19)$ and $(25)$. The center of $\mathcal{U}_{q}(\mathfrak{n})$ is the polynomial algebra $\mathbb{k}\left[z, y^{2}\right]$. The center of $\operatorname{Frac}\left(\mathcal{U}_{q}(\mathfrak{n})\right)$ is the purely transcendental extension $\mathbb{k}\left(y, z^{2}\right)$.

Proof. With the notations used in the proof of the proposition, the skewfield of fractions $F:=$ Frac $N_{q}=$ Frac $N_{q}^{\prime}$ is the skewfield of rational functions $F=\mathbb{k}(z, y)(t ; \gamma)(x ; \sigma)$ where the automorphisms $\gamma$ and $\sigma$ are defined by $\gamma: z \mapsto z, y \mapsto-y$ and $\sigma: z \mapsto z, y \mapsto-y, t \mapsto q^{-1} t$. The proof is then similar to that of corollary 2.3 .4 
3.2. Quantum enveloping algebra of the positive Borel subsuperalgebra of $\mathfrak{o s p}(1,4)$. We summarize all notations of part 3.1 .

Definition 3.2.1. The quantum enveloping algebra of the positive Borel subsuperalgebra $\mathfrak{b}$ of the Lie superalgebra $\mathfrak{o s p}(1,4)$ is the superalgebra $\mathcal{U}_{q}(\mathfrak{b})$ generated by $\mathcal{U}_{q}(\mathfrak{n})$ and the commutative Cartan subsuperalgebra $\mathbb{k}\left[k_{1}^{ \pm 1}, k_{2}^{ \pm 1}\right]$, where $k_{1}$ and $k_{2}$ are even and act on the Chevalley generators $e_{1}, e_{2}$ by:

$$
k_{1} e_{1}=q^{2} e_{1} k_{1}, \quad k_{1} e_{2}=q^{-1} e_{2} k_{1}, \quad k_{2} e_{1}=q^{-1} e_{1} k_{2}, \quad k_{2} e_{2}=q e_{2} k_{2},
$$

see for instance [26, 27, 28].

Proposition 3.2.2. The superalgebra $\mathcal{U}_{q}(\mathfrak{b})$ is strongly s-rationally equivalent to the q-polynomial superalgebra represented by the diagram:

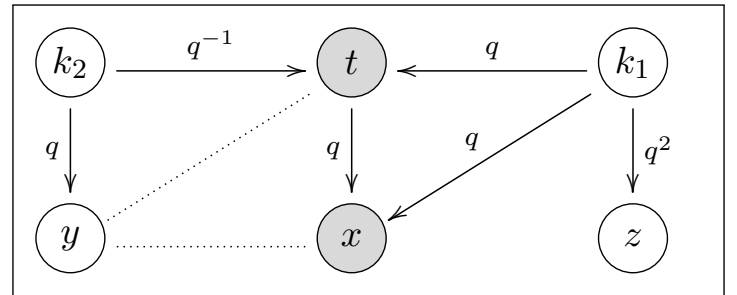

Proof. Starting from 20, we obtain a description of $B_{q}:=\mathcal{U}_{q}(\mathfrak{b})$ as an iterated Ore extension:

$$
B_{q}=\mathbb{k}\left[e_{1}, y\right][x ; \sigma]\left[e_{2} ; \tau, \delta\right]\left[k_{1}^{ \pm 1}, \theta_{1}\right]\left[k_{2}^{ \pm 1}, \gamma_{2}\right]
$$

where the automorphisms $\theta_{1}$ and $\gamma_{2}$ correspond to the relations

$$
k_{1} y=y k_{1}, \quad k_{2} y=q y k_{2}, \quad k_{1} x=q x k_{1}, \quad k_{2} x=x k_{2}
$$

deduced from (18), (19) and (29). This action of $k_{1}$ and $k_{2}$ extends to the localization $N_{q}^{\prime}=\mathbb{k}[z]\left[t^{ \pm}\right]\left[x^{ \pm} ; \sigma\right][y ; \gamma]$ described by (28). By definitions 23) and (27) of $z$ and $t$, we have:

$$
k_{1} z=q^{2} z k_{1}, \quad k_{2} z=z k_{2} \quad k_{1} t=q t k_{1}, \quad k_{2} t=q^{-1} t k_{2} .
$$

Then the localized superalgebra

$$
B_{q}^{\prime}:=N_{q}^{\prime}\left[k_{1}^{ \pm 1}, \theta_{1}\right]\left[k_{2}^{ \pm 1}, \theta_{2}\right]
$$

is s-rationally equivalent to the $q$-polynomial superalgebra described by the diagram above, and the result follows from the application of corollary 1.1.3 to the multiplicative subset generated by $x, t, k_{1}, k_{2}$.

Up to further localizations by homogeneous elements, there are of course many ways to reduce the commutation diagram to more simple forms. In addition to the question of which ones are the most significant, some reductions may facilitate calculations to show some properties, such as the determination of the center in the following corollary.

Corollary 3.2.3. If $q$ is not a root of one, the center of $\mathcal{U}_{q}(\mathfrak{b})$ and the center of $\operatorname{Frac}\left(\mathcal{U}_{q}(\mathfrak{b})\right)$ are equal to $\mathbb{k}$. 
Proof. With the notations used in the proof of the preceding proposition, we introduce using lemma 1.1.1 the localization $B_{q}^{\prime \prime}$ of $B_{q}^{\prime}$ with respect to the powers of the even element $z$. We introduce in $B_{q}^{\prime \prime}$ the homogeneous changes of variables: $x^{\prime}:=k_{2}^{-1} x$ and $t^{\prime}:=z^{-1} x t$, then $z^{\prime}=\left(x^{\prime}\right)^{-2} z$ and $y^{\prime}=t^{\prime} k_{2}^{-1} y$. By straightforward calculations, we check that the superalgebra $B_{q}^{\prime \prime}$ is s-rationally equivalent to the $q$-polynomial superalgebra represented by the diagram:

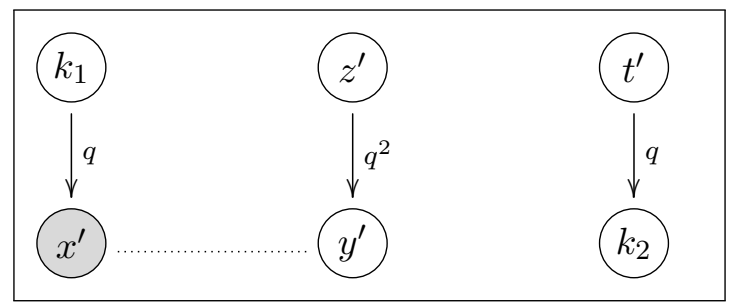

The skewfield of fractions $F:=$ Frac $B_{q}=$ Frac $B_{q}^{\prime \prime}$ can be canonically embedded in an iterated skewfield of Laurent series

$$
\bar{F}=\mathbb{k}\left(\left(x^{\prime}\right)\right)\left(\left(y^{\prime} ; \sigma^{\prime}\right)\right)\left(\left(z^{\prime} ; \tau^{\prime}\right)\right)\left(\left(t^{\prime}\right)\right)\left(\left(k_{1} ; \theta_{1}\right)\right)\left(\left(k_{2} ; \theta_{2}\right)\right)
$$

for suitable automorphism corresponding to the above commutation picture. A nonzero element $f \in F$ is central if and only if any monomial

$$
f_{n}=\lambda_{n} x^{\prime n_{1}} y^{\prime n_{2}} z^{\prime n_{3}} t^{\prime n_{4}} k_{1}^{n_{5}} k_{2}^{n_{6}}, n=\left(n_{1}, \ldots, n_{6}\right) \in \mathbb{Z}^{6}, \lambda_{n} \in \mathbb{k}, \lambda_{n} \neq 0
$$

in the development of $f$ in $\bar{F}$ commutes with each variable $x^{\prime}, y^{\prime}, z^{\prime}, t^{\prime}, k_{1}$ and $k_{2}$. We have $f_{n} k_{1}=k_{1} f_{n}$ if and only if $q^{n_{1}}=1$ or equivalently $n_{1}=0$. Similarly $f_{n} k_{2}=k_{2} f_{n}$ is equivalent to $n_{4}=0$. We deduce step by step that $f \in \mathbb{k}$. We conclude that $Z\left(\operatorname{Frac} B_{q}\right)=\mathbb{k}$ and then $Z\left(\mathcal{U}_{q}(\mathfrak{b})\right)=\mathbb{k}$.

\subsection{Quantum enveloping algebra of a positive parabolic subsuper-} algebra of $\mathfrak{o s p}(1,4)$. We summarize all notations of the parts 3.1 and 3.2 .

Definition 3.3.1. The quantum enveloping algebra of the positive parabolic subsuperalgebra $\mathfrak{p}$ of the Lie superalgebra $\mathfrak{o s p}(1,4)$ is the superalgebra $\mathcal{U}_{q}(\mathfrak{p})$ generated by $\mathcal{U}_{q}(\mathfrak{b})$ and the odd generator $f_{2}$ acting on the Chevalley generators $e_{1}, e_{2}$ and the Cartan generators $k_{1}^{ \pm}, k_{2}^{ \pm}$by:

$$
\begin{array}{ll}
f_{2} e_{1}=e_{1} f_{2}, & f_{2} e_{2}=-e_{2} f_{2}+\frac{1}{q-q^{-1}}\left(k_{2}-k_{2}^{-1}\right), \\
f_{2} k_{1}=q^{-1} k_{1} f_{2}, & f_{2} k_{2}=q k_{2} f_{2},
\end{array}
$$

see for instance [26, 27, 28].

Theorem 3.3.2. The superalgebra $\mathcal{U}_{q}(\mathfrak{p})$ is s-rationally equivalent to the q-polynomial superalgebra represented by the diagram: 


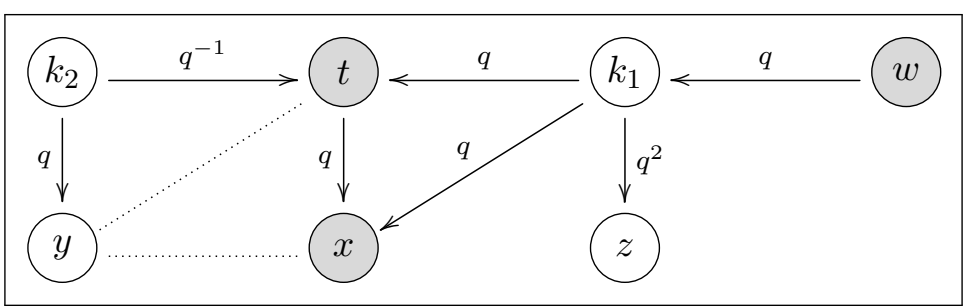

Proof. We denote $P_{q}:=\mathcal{U}_{q}(\mathfrak{p})$. By direct calculations, we deduce from (34) the action of $f_{2}$ on the elements $x, u, z$ of $N_{q}$ introduced in 218, 22, 23):

$$
f_{2} x=-x f_{2}+q^{-1} e_{1} k_{2}, \quad f_{2} t=-t f_{2}+k_{2} t^{2}, \quad f_{2} z=z f_{2} .
$$

The relation for the generator $y$ defined by 19 is more complicated:

$$
f_{2} y=y f_{2}-\left(1+q^{-1}\right) e_{2} e_{1} k_{2}+\frac{2 q+1+q^{-1}}{q-q^{-1}} x k_{2}+\frac{1+q}{q-q^{-1}} x k_{2}^{-1} .
$$

Starting with the description (30) of $B_{q}$, we have the Ore extension $P_{q}=$ $B_{q}\left[f_{2} ; \alpha, d\right]$ where the automorphism $\alpha$ and the $\alpha$-derivation $d$ of $B_{q}$ correspond to relations (34), (35) and (36). By localization, we have similarly $P_{q}^{\prime}:=B_{q}^{\prime}\left[f_{2} ; \alpha, d\right]$ where $B_{q}^{\prime}$ is defined by (33) and $(28)$. In order to simplify the first relation in (35), we can replace $f_{2}$ by:

$$
f_{2}^{\prime}:=(q+1) f_{2}-e_{1} x^{-1} k_{2}=(q+1) f_{2}-k_{2} t,
$$

which satisfies:

$$
f_{2}^{\prime} x=-x f_{2}^{\prime}, \quad f_{2}^{\prime} z=z f_{2}^{\prime}, \quad f_{2}^{\prime} t=-t f_{2}^{\prime}, \quad f_{2}^{\prime} k_{2}=q k_{2} f_{2}^{\prime} .
$$

Relation (36) becomes:

$$
f_{2}^{\prime} y=y f_{2}^{\prime}+\frac{q(q+1)}{q-1} k_{2}^{-1} x+\frac{1}{1-q} x^{-1} z k_{2} .
$$

Then $P_{q}^{\prime}=B_{q}^{\prime}\left[f_{2}^{\prime} ; \alpha^{\prime} ; d^{\prime}\right]$ where the automorphism $\alpha^{\prime}$ and the $\alpha^{\prime}$-derivation $d^{\prime}$ correspond to these transformed relations. We observe that (38) and (39) allow in particular to consider between $N_{q}^{\prime}$ defined by 28) and $P_{q}^{\prime}$ the intermediate superalgebra

$$
H_{q}:=\mathbb{k}[z]\left[t^{ \pm}\right]\left[x^{ \pm} ; \sigma\right]\left[k_{2}^{ \pm} ; \theta_{2}\right][y ; \gamma]\left[f_{2}^{\prime} ; \alpha^{\prime} ; d^{\prime}\right] .
$$

A key observation is that in this description $\alpha^{\prime}(z)=z=\gamma^{-1}(z), \alpha^{\prime}(t)=$ $-t=\gamma^{-1}(t), \alpha^{\prime}(x)=-x=\gamma^{-1}(x), \alpha^{\prime}\left(k_{2}\right)=q k_{2}=\gamma^{-1}\left(k_{2}\right)$. Hence the automorphism $\alpha^{\prime}$ is equal to $\gamma^{-1}$ on $\mathbb{k}[z]\left[t^{ \pm}\right]\left[x^{ \pm} ; \sigma\right]\left[k_{2}^{ \pm} ; \theta_{2}\right]$ and satisfies $\alpha^{\prime}(y)=y$. Then we can apply the method introduced in section 2.1 of [18] and write the value of the $\gamma^{-1}$-derivation $d^{\prime}$ in $y$ under the form:

$$
d^{\prime}(y)=\frac{q(q+1)}{q-1} k_{2}^{-1} x+\frac{1}{1-q} x^{-1} z k_{2}=h-\gamma(h)
$$

with notation:

$$
h:=\frac{q}{1-q} k_{2}^{-1} x-\frac{q}{(1-q)^{2}} x^{-1} z k_{2} .
$$

By construction, the odd element:

$$
w:=y f_{2}^{\prime}+\gamma(h)=f_{2}^{\prime} y+h
$$


commutes with $y$. Moreover it follows from the commutation relations in $B_{q}^{\prime}$ (see diagram in proposition 3.2 .2 that $h$ commutes in $B_{q}^{\prime}$ with $x, y, z, t$ and $k_{2}$. Then by (38) the same is true for $w$. Finally we can by (43) replace the generator $f_{2}^{\prime}$ by the generator $w$ in the localization $H_{q}^{\prime}$ of $H_{q}$ with respect to the powers of $y$, and we obtain:

$$
H_{q}^{\prime}:=\mathbb{k}[z]\left[t^{ \pm}\right]\left[x^{ \pm} ; \sigma\right]\left[k_{2}^{ \pm} ; \theta_{2}\right]\left[y^{ \pm} ; \gamma\right][w] .
$$

The next step consists in introducing the localization $P_{q}^{\prime \prime}$ of $P_{q}^{\prime}$ with respect to the powers of $y$. We obtain:

$$
P_{q}^{\prime \prime}:=\mathbb{k}[z]\left[t^{ \pm}\right]\left[x^{ \pm} ; \sigma\right]\left[k_{2}^{ \pm} ; \theta_{2}\right]\left[y^{ \pm} ; \gamma\right][w]\left[k_{1}^{ \pm} ; \theta_{1}\right] .
$$

It is clear by (32), (34) and (37) that $k_{1} f_{2}^{\prime}=q^{-1} f_{2}^{\prime} k_{1}$ and then $k_{1} w=q^{-1} w k_{1}$ by (43). We conclude that the superalgebra $P_{q}^{\prime \prime}$ is s-rationally equivalent to the $q$-polynomial superalgebra generated over $\mathbb{k}$ by the even generators $y, z, k_{1}, k_{2}$ and the odd generators $x, t, w$. Using corollary 1.1.3, the proof is complete.

Corollary 3.3.3. We suppose that $q$ is not a root of one. Then the center of $\mathcal{U}_{q}(\mathfrak{p})$ is $\mathbb{k}$ and the center of Frac $\left(\mathcal{U}_{q}(\mathfrak{p})\right)$ is the purely transcendental extension $\mathbb{k}\left(z^{\prime}\right)$, where $z^{\prime}:=w^{-2} z$ with $z, w$ defined by (25) and (43).

Proof. It is clear by (45) and the associated commutation picture that any central element $f \neq 0$ in the superalgebra $P_{q}^{\prime \prime}$ is a finite sum of central monomials $f_{n}$ of the form:

$$
f_{n}=\lambda_{n} x^{n_{1}} y^{n_{2}} z^{n_{3}} t^{n_{4}} k_{1}^{n_{5}} k_{2}^{n_{6}} w^{n_{7}}, n=\left(n_{1}, \ldots, n_{7}\right) \in \mathbb{Z}^{7}, \lambda_{n} \in \mathbb{k}, \lambda_{n} \neq 0,
$$

where $n_{3}$ and $n_{7}$ are nonnegative. By writing successively that $f_{n}$ commutes with $z, x, y, t$ and $k_{2}$ (in this order) we obtain that the integers $n_{5}, n_{4}, n_{6}$ $n_{1}$ and $n_{2}$ are zero. Hence $f_{n}=\lambda_{n} z^{n_{3}} w^{n_{7}}$. The commutation $k_{1} f_{n}=f_{n} k_{1}$ implies $2 n_{3}+n_{7}=0$, then $n_{3}=n_{7}=0$. We conclude that the center of $P_{q}^{\prime \prime}$ is $\mathbb{k}$ and so the center of $P_{q}$ is $\mathbb{k}$. It is clear that in the localization $P_{q}^{\prime \prime \prime}$ of $P_{q}^{\prime \prime}$ with respect to the powers of $w$, we can replace the generator $z$ by the central generator $z^{\prime}:=w^{-2} z$ to conclude that the center of $P_{q}^{\prime \prime \prime}$ is $\mathbb{k}\left[z^{\prime}\right]$. The center of Frac $\left(P_{q}\right)$ is then obtained by embedding Frac $P_{q}=$ Frac $P_{q}^{\prime \prime \prime}$ in a suitable skewfield of Laurent series as in the proof of 3.2 .3

\section{REFERENCES}

[1] J. Alev and F. Dumas. Sur le corps des fractions de certaines algèbres quantiques. J. Algebra, 170(1):229-265, 1994.

[2] Jacques Alev and François Dumas. On enveloping skew fields of some Lie superalgebras. J. Algebra Appl., 15(4):1650071, 16, 2016.

[3] Jacques Alev and François Dumas. Enveloping skewfields of the nilpotent positive part and the Borel subsuperalgebra of $\mathfrak{o s p}(1,2 n)$. In Rings, modules and codes, volume 727 of Contemp. Math., pages 7-23. Amer. Math. Soc., Providence, RI, 2019.

[4] D. Arnaudon and M. Bauer. Scasimir operator, scentre and representations of $U_{q}(\operatorname{osp}(1 \mid 2))$. Lett. Math. Phys., 40(4):307-320, 1997.

[5] Marc Aubry and Jean-Michel Lemaire. Zero divisors in enveloping algebras of graded Lie algebras. J. Pure Appl. Algebra, 38(2-3):159-166, 1985. 
[6] I. N. Balaba, A. L. Kanunnikov, and A. V. Mikhalëv. Quotient rings of graded associative rings. I. Fundam. Prikl. Mat., 17(2):3-74, 2011/12.

[7] Erazm J. Behr. Enveloping algebras of Lie superalgebras. Pacific J. Math., 130(1):925, 1987.

[8] Rikard Bøgvad. Some elementary results on the cohomology of graded Lie algebras. In Algebraic homotopy and local algebra (Luminy, 1982), volume 113 of Astérisque, pages 156-166. Soc. Math. France, Paris, 1984.

[9] Ken A. Brown and Ken R. Goodearl. Lectures on algebraic quantum groups. Advanced Courses in Mathematics. CRM Barcelona. Birkhäuser Verlag, Basel, 2002.

[10] Pierre Deligne and John W. Morgan. Notes on supersymmetry (following Joseph Bernstein). In Quantum fields and strings: a course for mathematicians, Vol. 1, 2 (Princeton, NJ, 1996/1997), pages 41-97. Amer. Math. Soc., Providence, RI, 1999.

[11] Jacques Dixmier. Algèbres enveloppantes. Gauthier-Villars Éditeur, Paris-BrusselsMontreal, Que., 1974. Cahiers Scientifiques, Fasc. XXXVII.

[12] François Dumas. Sous-corps de fractions rationnelles des corps gauches de séries de Laurent. In Topics in invariant theory (Paris, 1989/1990), volume 1478 of Lecture Notes in Math., pages 192-214. Springer, Berlin, 1991.

[13] Vyacheslav Futorny and Jonas T. Hartwig. Solution of a $q$-difference Noether problem and the quantum Gelfand-Kirillov conjecture for $\mathfrak{g l}_{N}$. Math. Z., 276(1-2):1-37, 2014.

[14] I. M. Gelfand and A. A. Kirillov. Sur les corps liés aux algèbres enveloppantes des algèbres de Lie. Inst. Hautes Études Sci. Publ. Math., (31):5-19, 1966.

[15] K. R. Goodearl and J. T. Stafford. The graded version of Goldie's theorem. In Algebra and its applications (Athens, OH, 1999), volume 259 of Contemp. Math., pages 237240. Amer. Math. Soc., Providence, RI, 2000.

[16] K. R. Goodearl and R. B. Warfield, Jr. An introduction to noncommutative Noetherian rings, volume 16 of London Mathematical Society Student Texts. Cambridge University Press, Cambridge, 1989.

[17] Miloslav Havlíček, Jan Kotrbatý, Patrick Moylan, and Severin Pošta. Construction of representations of Poincaré group using Lie fields. J. Math. Phys., 59(2):021702, 23, 2018.

[18] David A. Jordan. A simple localization of the quantized Weyl algebra. J. Algebra, 174(1):267-281, 1995.

[19] Christian Kassel. Quantum groups, volume 155 of Graduate Texts in Mathematics. Springer-Verlag, New York, 1995.

[20] J. C. McConnell and J. C. Robson. Noncommutative Noetherian rings. Pure and Applied Mathematics (New York). John Wiley \& Sons, Ltd., Chichester, 1987. With the cooperation of L. W. Small, A Wiley-Interscience Publication.

[21] Susan Montgomery. Fixed rings of finite automorphism groups of associative rings, volume 818 of Lecture Notes in Mathematics. Springer, Berlin, 1980.

[22] Patrick Moylan. Localizations of $U_{q}(\mathfrak{s l}(2))$ and $U_{p}(\mathfrak{o s p}(1 \mid 2))$ associated with Euclidean and super Euclidean algebras. In Lie theory and its applications in physics, volume 111 of Springer Proc. Math. Stat., pages 369-379. Springer, Tokyo, 2014.

[23] Ian M. Musson. Some Lie superalgebras associated to the Weyl algebras. Proc. Amer. Math. Soc., 127(10):2821-2827, 1999.

[24] Ian M. Musson. Lie superalgebras and enveloping algebras, volume 131 of Graduate Studies in Mathematics. American Mathematical Society, Providence, RI, 2012.

[25] C. Năstăsescu and F. van Oystaeyen. Methods of graded rings, volume 1836 of Lecture Notes in Mathematics. Springer-Verlag, Berlin, 2004.

[26] T. D. Palev. Quantization of $U_{q}[\operatorname{osp}(1 / 2 n)]$ with deformed para-Bose operators. $J$. Phys. A, 26(21):L1111-L1116, 1993.

[27] T. D. Palev and J. Van der Jeugt. The quantum superalgebra $U_{q}[\operatorname{osp}(1 \mid 2 n)]$ : deformed para-Bose operators and root of unity representations. J. Phys. A, 28(9):2605-2616, 1995. 
[28] R. B. Zhang. Finite-dimensional representations of $U_{q}(\operatorname{osp}(1 / 2 n))$ and its connection with quantum so(2n+1). Lett. Math. Phys., 25(4):317-325, 1992.

(J. Alev) Université de Reims, Laboratoire de Mathématiques (FRE 2011 CNRS), Moulin de la Housse, B.P. 1039, 51687 Reims cedex 2 (France)

Email address: jacques.alev@univ-reims.fr

(F. Dumas) Université Clermont Auvergne, Laboratoire de Mathématiques Blaise Pascal (UMR 6620 - CNRS), 3 Place VAsarely, CS 60026, 63178 Aubière CEDEX (FRANCE)

Email address: Francois.Dumas@uca.fr 